\title{
Fuzzy Prime Ideals of ADL's
}

\author{
Chigurupalli Santhi Sundar Raj, Natnael Teshale Amare and Uppasetti Madana Swamy
}

\begin{abstract}
In this paper the concept of prime $L$-fuzzy ideals and $L$-fuzzy prime ideals of an ADL $A$ with truth values in a complete lattice $L$ satisfying the infinite meet distributive law are introduced. All prime $L$-fuzzy ideals of a given ADL $A$ are determined by establishing a one-to- one correspondence between prime $L$-fuzzy ideals of an ADL $A$ and the pairs $(P, \alpha)$, where $P$ is a prime ideal of $A$ and $\alpha$ is a prime element in $L$. Also, here minimal prime $L$-fuzzy ideals and $L$-fuzzy minimal prime ideals of an ADL $A$ are introduced and characterized.

Index Terms-Almost Distributive Lattice (ADL), complete lattice, L-fuzzy minimal prime ideal L-fuzzy prime ideal, minimal prime L-fuzzy ideal, prime L-fuzzy ideal.
\end{abstract}

\section{INTRODUCTION}

A fuzzy subset of a set $X$ is a function from $X$ into $I=$ $[0,1]$, as in [1]. J.A. Goguen [2] explored, generalized and continued the work of L.A. Zadeh and realized that the unit interval $[0,1]$ is not sufficient to take the truth values of general fuzzy statements. Wang-Jing Liu [3] introduced the notion of a fuzzy ideal of a ring in the case when $L=[0,1]$ of real numbers and T.K. Mukherjee and M.K. Sen [4] introduced the notion of a fuzzy prime ideal and continued the study of fuzzy ideals. U.M. Swamy and K.L.N. Swamy [5] introduced the concept of fuzzy prime ideal of a ring with truth values in a complete lattice satisfying the infinite meet distributive law.

The concept of prime ideal of an Almost Distributive Lattice was introduced by U.M. Swamy and G.C. Rao, in 1981 [6]. U.M. Swamy, Ch. Santhi Sundar Raj and Natnael Teshale A [7] have introduced the notion of $L$-fuzzy ideals of an ADL with the truth values in a complete lattice $L$ satisfying the infinite meet distributive law.

In this paper, we introduce and study prime $L$-fuzzy ideals and $L$-fuzzy prime ideals of an $\operatorname{ADL} A$, where $L$ is a complete lattice satisfying the infinite meet distributive law. Also, in this paper we introduce minimal prime $L$-fuzzy ideals and $L$-fuzzy minimal prime ideals of an ADL $A$.

\section{PREliminaries}

First we give necessary definitions and results mostly taken from [6] and [7] which will be used in the later text.

Definition 2.1: An algebra $A=(A, \wedge, \vee, 0)$ of type $(2,2,0)$ is called an Almost Distributive Lattice (abbreviated as ADL) if it satisfies the following conditions for all $a, b$ and $c \in A$.

1) $0 \wedge a=0$

Manuscript received December 19, 2017; accepted May 10, 2018.

Ch. Santhi Sundar Raj and A. Natnael Teshale are with the Department of Engineering Mathematics, Andhra University, Visakhapatnam- 530003, A.P., India. E-mail: santhisundarraj@yahoo.com

A. Natnael Teshale is with the Department of Mathematics, University of Gondar, Ethiopia. E-mail: yenatnaelau@yahoo.com

U.M. Swamy is with the Department of Mathematics, Andhra University, Visakhapatnam- 530003, A.P., India. E-mail: umswamy@yahoo.com
2) $a \vee 0=a$

3) $a \wedge(b \vee c)=(a \wedge b) \vee(a \wedge c)$

4) $a \vee(b \wedge c)=(a \vee b) \wedge(a \vee c)$

5) $(a \vee b) \wedge c=(a \wedge c) \vee(b \wedge c)$

6) $(a \vee b) \wedge b=b$.

Any bounded below distributive lattice is an ADL, where 0 is the smallest element. Any nonempty set $X$ can be made into an ADL by fixing an arbitrarily chosen element 0 in $X$ and by defining the binary operations $\wedge$ and $\vee$ on $X$ by

$$
a \wedge b=\left\{\begin{array}{ll}
0, & \text { if } a=0 \\
b, & \text { if } a \neq 0
\end{array} \quad \text { and } \quad a \vee b= \begin{cases}b, & \text { if } a=0 \\
a, & \text { if } a \neq 0\end{cases}\right.
$$

This $\operatorname{ADL}(X, \wedge, \vee, 0)$ is called a discrete ADL.

Definition 2.2: Let $A=(A, \wedge, \vee, 0)$ be an ADL. For any $a$ and $b \in A$, define $a \leq b$ if $a=a \wedge b(\Leftrightarrow a \vee b=b)$. Then $\leq$ is a partial order on $A$ with respect to which 0 is the smallest element in $A$.

Theorem 2.3: The following hold for any $a, b$ and $c$ in an ADL $A$.

(1) $a \wedge 0=0=0 \wedge a$ and $a \vee 0=a=0 \vee a$

(2) $a \wedge a=a=a \vee a$

(3) $a \wedge b \leq b \leq b \vee a$

(4) $a \wedge b=a \Leftrightarrow a \vee b=b$

(5) $a \wedge b=b \Leftrightarrow a \vee b=a$

(6) $(a \wedge b) \wedge c=a \wedge(b \wedge c)$ (i.e., $\wedge$ is associative)

(7) $a \vee(b \vee a)=a \vee b$

(8) $a \leq b \Rightarrow a \wedge b=a=b \wedge a(\Leftrightarrow a \vee b=b=b \vee a)$

(9) $(a \wedge b) \wedge c=(b \wedge a) \wedge c$

(10) $(a \vee b) \wedge c=(b \vee a) \wedge c$

(11) $a \wedge b=b \wedge a \Leftrightarrow a \vee b=b \vee a$

(12) $a \wedge b=\inf \{a, b\} \Leftrightarrow a \wedge b=b \wedge a \Leftrightarrow a \vee b=\sup \{a, b\}$.

An element $m \in A$ is said to be maximal if, for any $x \in$ $A, m \leq x$ implies $m=x$. It can be easily observed that $m$ is maximal if and only if $m \wedge x=x$ for all $x \in A$.

Definition 2.4: Let $I$ be a non empty subset of an ADL $A$. Then $I$ is called an ideal of $A$ if $a, b \in I \Rightarrow a \vee b \in I$ and $a \wedge x \in I$ for all $x \in A$.

As a consequence, for any ideal $I$ of $A, x \wedge a \in I$ for all $a \in I$ and $x \in A$. For any $S \subseteq A$, the smallest ideal of $A$ containing $S$ is called the ideal generated by $S$ in $A$ and is denoted by $(S]$. It is known that

$$
(S]=\left\{\left(\bigvee_{i=1}^{n} x_{i}\right) \wedge a \mid n \geq 0, x_{i} \in S \text { and } a \in A\right\} .
$$

when $S=\{x\}$, we write $(x]$ for $(\{x\}]$. Note that $(x]=\{x \wedge a \mid a \in A\}$.

Definition 2.5: An $L$-fuzzy subset $\lambda$ of $X$ is a mapping from $X$ into $L$, where $L$ is a complete lattice satisfying the infinite meet distributive law. If $L$ is the unit interval $[0,1]$ of real numbers, then these are the usual fuzzy subsets of $X$. 
For any $\alpha \in L$, the set $\lambda_{\alpha}=\{x \in X: \alpha \leq \lambda(x)\}$ is called the $\alpha$-cut of $\lambda$.

Definition 2.6: An $L$-fuzzy subset $\lambda$ of $A$ is said to be an $L$-fuzzy ideal of $A$, if $\lambda(0)=1$ and $\lambda(x \vee y)=\lambda(x) \wedge \lambda(y)$, for all $x, y \in A$.

Lemma 2.7: Let $\lambda$ be an $L$-fuzzy ideal of $A, S$ a non-empty subset of $A$ and $x, y \in A$. Then we have the following.

(1) $x \wedge y=y$ and $y \wedge x=x \Longrightarrow \lambda(x)=\lambda(y)$

(2) $\lambda(x \wedge y)=\lambda(y \wedge x)$

(3) $x \in(S] \Longrightarrow \lambda(x) \geq \bigwedge_{i=1}^{n} \lambda\left(a_{i}\right)$ for some $a_{1}, a_{2}, \ldots, a_{n} \in S$

(4) $x \in(y] \Longrightarrow \lambda(x) \geq \lambda(y)$

(5) If $m$ is a maximal element in $A$ then $\lambda(m) \leq \lambda(x)$, for all $x$

(6) $\lambda(m)=\lambda(n)$ for all maximal elements $m$ and $n$ in $A$.

Theorem 2.8: The set of all $L$-fuzzy ideals of $A$ is a complete distributive lattice, in which the supremum $\bigvee_{i \in \Delta} \lambda_{i}$ and infimum $\bigwedge_{i \in \Delta} \lambda_{i}$ of any family

$\left\{\lambda_{i}: i \in \Delta\right\}$ of $L$-fuzzy ideals of $A$ are given by

$$
\begin{aligned}
\left(\bigvee_{i \in \Delta} \lambda_{i}\right)(x) & =\bigvee\left\{\bigwedge_{a \in F}\left(\bigvee_{i \in \Delta} \lambda_{i}(a)\right): x \in(F], F \subset \subset A\right\} \\
\text { and }\left(\bigwedge_{i \in \Delta} \lambda_{i}\right)(x) & =\bigwedge_{i \in \Delta} \lambda_{i}(x)
\end{aligned}
$$

\section{PRIME $L$-FuZZY IDEALS}

Let us recall from [6] that a proper ideal $P$ of an ADL $A$ is said to be prime if for any $x, y \in A, x \wedge y \in P$ implies that $x \in P$ or $y \in P$; (equivalently, for any ideals $I$ and $J$ of $A$, $I \cap J \subseteq P \Rightarrow I \subseteq P$ or $J \subseteq P$.)

The following definition is analogous to that of a prime ideal of $A$. Here after $A$ stands for an ADL with a maximal element. An $L$-fuzzy ideal $\lambda$ of $A$ is called proper if $\lambda(x) \neq 1$ for some $x \in A$.

Definition 3.1: A proper $L$-fuzzy ideal $\lambda$ of $A$ is called a prime $L$-fuzzy ideal if for any $L$-fuzzy ideals $v$ and $\mu$ of $A$, $v \wedge \mu \leq \lambda$ implies either $v \leq \lambda$ or $\mu \leq \lambda$.

An element $x \neq 1$ in $L$ is called prime if for any $a, b \in L$ $a \wedge b \leq x$ implies either $a \leq x$ or $b \leq x$.

Now, we determine all prime $L$-fuzzy ideals of $A$ by establishing a correspondence between prime $L$-fuzzy ideals and pairs $(I, \alpha)$, where $I$ is a prime ideal of $A$ and $\alpha$ is a prime element in $L$. First, we recall from [7] that for any ideal $I$ of $A$ and $\alpha \in L$, the $L$-fuzzy ideal $\alpha_{I}$ of $A$ defined by

$$
\alpha_{I}(x)= \begin{cases}1 & \text { if } x \in I \\ \alpha & \text { if } x \notin I .\end{cases}
$$

and that $\alpha_{I}$ is called the $\alpha$-level $L$-fuzzy ideal correspondence to $I$.

Theorem 3.2: Let $I$ be an ideal of an ADL $A$ and $\alpha \in L$. Then $\alpha_{I}$ is a prime $L$-fuzzy ideal of $A$ if and only if $I$ is a prime ideal of $A$ and $\alpha$ is a prime element in $L$.

Proof: Suppose that $\alpha_{I}$ is a prime $L$-fuzzy ideal of $A$. Since $\alpha_{I}$ is proper, $\alpha_{I}(x) \neq 1$, for some $x \in A$. Therefore $x \notin I$ and hence $I \subsetneq A$. If $J$ and $K$ are ideals of $A$ such that $J \cap K \subseteq I$. Then $\alpha_{J} \wedge \alpha_{K}=\alpha_{J \wedge K} \leq \alpha_{I}$ and hence $\alpha_{J} \leq \alpha_{I}$ or $\alpha_{K} \leq \alpha_{I}$, so that $J \subseteq I$ or $K \subseteq I$. Therefore, $I$ is a prime ideal of $A$. Also, for any $\gamma, \beta \in L$,

$$
\begin{aligned}
\gamma \wedge \beta \leq \alpha & \Rightarrow(\gamma \wedge \beta)_{I} \leq \alpha_{I} \\
& \Rightarrow \gamma_{I} \wedge \beta_{I} \leq \alpha_{I} \\
& \Rightarrow \gamma_{I} \leq \alpha_{I} \text { or } \beta_{I} \leq \alpha_{I} \\
& \Rightarrow \gamma \leq \alpha \text { or } \beta \leq \alpha .
\end{aligned}
$$

Therefore, $\alpha$ is a prime element in $L$.

Conversely, suppose that $I$ is a prime ideal of $A$ and $\alpha$ is a prime element in $L$. Since $I$ is proper and $\alpha \neq 1, \alpha_{I}$ is clearly a proper $L$-fuzzy ideal of $A$. Let $\lambda$ and $\mu$ be any $L$-fuzzy ideals of $A$ such that $\lambda \not \leq \alpha_{I}$ and $\mu \not \leq \alpha_{I}$. Then there exists $x, y \in A$ such that $\lambda(x) \not \alpha_{I}(x)$ and $\mu(y) \not \leq \alpha_{I}(y)$. This implies that $\alpha_{I}(x)=\alpha=\alpha_{I}(y)$ (otherwise, $\alpha_{I}(x)=1 \geq \lambda(x)$ and $\alpha_{I}(y)=$ $1 \geq \mu(y))$ and hence $x \notin I$ and $y \notin I$. Since $I$ is a prime ideal, $x \wedge y \notin I$. Also, since $\alpha$ is prime and $\lambda(x) \not \leq \alpha$ and $\mu(y) \not \leq \alpha$, we have that $\lambda(x) \wedge \mu(y) \not \leq \alpha$.

Now, $(\lambda \wedge \mu)(x \wedge y)=\lambda(x \wedge y) \wedge \mu(x \wedge y) \geq \lambda(x) \wedge \mu(y)$ (since $\lambda$ and $\mu$ are antitones) and hence $(\lambda \wedge \mu)(x \wedge y) \not \leq \alpha=$ $\alpha_{I}(x \wedge y)$ so that, $(\lambda \wedge \mu) \not \alpha_{I}$. Hence, $\alpha_{I}$ is a prime $L$-fuzzy ideal of $A$.

Theorem 3.3: A proper $L$-fuzzy ideal $\lambda$ of $A$ is prime if and only if the following are satisfied.

(1) $\lambda$ is two valued

(2) $\lambda(m)$ is a prime element in $L$, for any maximal element $m$ in $A$

3) $\lambda_{1}$ is a prime ideal of $A$.

Proof: Suppose that $\lambda$ is a prime $L$-fuzzy ideal of $A$.

(1): Suppose $\lambda$ assumes more than two values. Then there exists $x, y \in A$ and $\alpha \neq \beta \in L-\{1\}$ such that $\lambda(x)=\alpha, \lambda(y)=$ $\beta$ and $\lambda(0)=1$. Now, define $L$-fuzzy subsets $v$ and $\mu$ of $A$ as follows:

$$
v(z)=\left\{\begin{array}{ll}
1 & \text { if } z \in(x] \\
0 & \text { if } z \notin(x]
\end{array} \text { and } \mu(z)= \begin{cases}1 & \text { if } z=0 \\
\alpha & \text { if } z \neq 0 .\end{cases}\right.
$$

Then, clearly $v=0_{(x]}$ and $\mu=\alpha_{(0]}$ and hence $v$ and $\mu$ are $L$-fuzzy ideals. Also,for $z=0 \Rightarrow(v \wedge \mu)(0)=v(0) \wedge \mu(0)=1 \wedge 1=1=\lambda(0)$.

$0 \neq z \in(x] \Rightarrow v(z) \wedge \mu(z)=1 \wedge \alpha=\alpha=\lambda(x) \leq \lambda(z)$ (since $\lambda$ is an antitone and $z \wedge x \leq x$, we have

$\lambda(x) \leq \lambda(z \wedge x)=\lambda(x \wedge z)=\lambda(z))$

and $z \notin(x] \Rightarrow v(z) \wedge \mu(z)=0 \wedge \alpha=0 \leq \lambda(z)$. Therefore, $v \wedge$ $\mu \leq \lambda$. Since $\lambda$ is prime, $v \leq \lambda$ or $\mu \leq \lambda$. But $v \not \leq \lambda$ (since $v(x)=1, \lambda(x)=\alpha$ and $1 \neq \alpha)$.

Therefore, $\mu \leq \lambda$. In particular, $\mu(y) \leq \lambda(y) \neq \lambda(0)$, we get that $y \neq 0$ and $\alpha=\mu(y)=\beta$, which is a contradiction.

(2): Let $m$ be a maximal element in $A$. Since $\lambda$ is proper, $\lambda(x) \neq 1$, for some $x \in A$ and hence $\lambda(m) \neq \lambda(0)=1$ $(\lambda(m)=1 \Rightarrow \lambda(m \vee x)=1 \Rightarrow \lambda(m) \wedge \lambda(x)=1 \Rightarrow \lambda(x)=1$. )

Let $\alpha$ and $\beta \in L$ such that $\alpha \wedge \beta \leq \lambda(m)$. Define $v$ and $\mu$ of $A$ as:

$$
v(x)=\left\{\begin{array}{ll}
1 & \text { if } x=0 \\
\alpha & \text { if } x \neq 0
\end{array} \quad \text { and } \quad \mu(x)= \begin{cases}1 & \text { if } x=0 \\
\beta & \text { if } x \neq 0 .\end{cases}\right.
$$

Then, it can be easily proved that $v$ and $\mu$ are $L$-fuzzy ideals of $A$ and $v \wedge \mu \leq \lambda$. Since $\lambda$ is prime, $v \leq \lambda$ or $\mu \leq \lambda$, inparticular, $v(m) \leq \lambda(m)$ or $\mu(m) \leq \lambda(m)$. Therefore, $\alpha \leq$ 
$\lambda(m)$ or $\beta \leq \lambda(m)$ and hence $\lambda(m)$ is prime.

(3): Let $I=\{x \in A: \lambda(x)=1\}$. Clearly, $I$ is a proper ideal of $A$, since $\lambda$ is proper. Let $\alpha$ be the other value of $\lambda$. Then

$$
\lambda(x)= \begin{cases}1 & \text { if } x \in I \\ \alpha & \text { if } x \notin I\end{cases}
$$

and hence $\lambda=\alpha_{I}$. By theorem 3.2, I is prime.

Conversely suppose that $\lambda$ is an $L$-fuzzy ideal of $A$ satisfying the conditions (1),(2) and (3). By (1), there exists $\alpha(\neq 1) \in$ $L$ such that $\lambda(x)=\alpha$, for each $x \in A-\{0\}$. Then for any maximal element $m$ of $A, \lambda(m)=\alpha$. By (2), $\alpha$ is prime. Let $I=\{x \in A: \lambda(x)=1\}$. Then $I$ is a prime ideal of $A$ (by (3)). Therefore, $\lambda=\alpha_{I}$ and hence $\lambda$ is a prime $L$-fuzzy ideal of $A$ (by theorem 3.2).

The results 3.2 and 3.3 yield the following.

Theorem 3.4: Let $\lambda$ be an $L$-fuzzy subset of $A$. Then $\lambda$ is a prime $L$-fuzzy ideal of $A$ if and only if there exists a prime ideal $P$ of $A$ and a prime element $\alpha$ in $L$ such that $\lambda=\alpha_{P}$.

\section{L-FUZZY PRIME IDEALS}

In this section, we introduce the notion of an $L$-fuzzy prime ideal which is weaker than that of a prime $L$-fuzzy ideal.

Definition 4.1: A proper $L$-fuzzy ideal $\lambda$ of $A$ is called an $L$-fuzzy prime ideal of $A$ if for any $x, y \in A$,

$\lambda(x \wedge y)=\lambda(x)$ or $\lambda(y)$.

The following theorem gives a characterization of an $L$ fuzzy prime ideal.

Theorem 4.2: Let $\lambda$ be a proper $L$-fuzzy ideal of $A$. Then the following are equivalent to each other.

(1) for each $\alpha \in L, \lambda_{\alpha}=A$ or $\lambda_{\alpha}$ is a prime ideal of $A$

(2) $\lambda$ is an $L$-fuzzy prime ideal of $A$

(3) for any $x, y \in A, \lambda(x \wedge y) \leq \lambda(x) \vee \lambda(y)$ and either $\lambda(x) \leq$ $\lambda(y)$ or $\lambda(y) \leq \lambda(x)$.

Proof: $(1) \Rightarrow(2)$ : Let $x, y \in A$ and $\alpha=\lambda(x \wedge y)$. Then $x \wedge y \in \lambda_{\alpha}$ and hence $x \in \lambda_{\alpha}$ or $y \in \lambda_{\alpha}$.

$$
\begin{aligned}
x \in \lambda_{\alpha} \Rightarrow & \lambda(x \wedge y)=\alpha \leq \lambda(x) \leq \lambda(x \wedge y) \\
& \Rightarrow \lambda(x \wedge y)=\lambda(x)
\end{aligned}
$$

Similarly, $y \in \lambda_{\alpha} \Rightarrow \lambda(x \wedge y)=\lambda(y)$.

(2) $\Rightarrow(3)$ : Let $x, y \in A$. Then, $\lambda(x \wedge y)=\lambda(x)$ or $\lambda(y)$.

$\lambda(y) \leq \lambda(x \wedge y)=\lambda(x)$.

Similarly, $\lambda(x \wedge y)=\lambda(y) \Rightarrow \lambda(x \wedge y) \leq \lambda(x) \vee \lambda(y)$ and (2) $I$ is a prime ideal of $A$ if and only if $\chi_{I}$ is an $L$-fuzzy prime $\lambda(x) \leq \lambda(y)$.

(3) $\Rightarrow(1)$ : Let $\alpha \in L$ be fixed. If $\lambda_{\alpha} \neq A$, then $\lambda_{\alpha}$ is a proper $(3)$ ideal of $A$. Also, for any $x, y \in A$,

$$
\begin{aligned}
x \wedge y \in \lambda_{\alpha} & \Rightarrow \alpha \leq \lambda(x \wedge y) \leq \lambda(x) \vee \lambda(y)=\lambda(x) \text { or } \lambda(y) \\
& \Rightarrow \alpha \leq \lambda(x) \text { or } \alpha \leq \lambda(y) \\
& \Rightarrow x \in \lambda_{\alpha} \text { or } y \in \lambda_{\alpha}
\end{aligned}
$$

Therefore, $\lambda_{\alpha}$ is prime.

Theorem 4.3: A prime $L$-fuzzy ideal of $A$ is an $L$-fuzzy prime ideal of $A$.

Proof: Let $\lambda$ be a prime $L$-fuzzy ideal of $A$. Then $\lambda=\alpha_{I}$ for some prime ideal $P$ of $A$ and $\alpha$ a prime element in $L$. Since $\alpha<1, \lambda$ is a proper.

Let $x, y \in A$. Then

$$
\begin{aligned}
x \wedge y \in I & \Rightarrow \lambda(x \wedge y)=1 \text { and } x \in I \text { or } y \in I \\
& \Rightarrow \lambda(x \wedge y)=1=\lambda(x) \text { or } \lambda(y)
\end{aligned}
$$

$$
\text { and } \begin{aligned}
x \wedge y \notin I \Rightarrow x & \notin I \text { and } y \notin I \\
& \Rightarrow \lambda(x \wedge y)=\alpha=\lambda(x)=\lambda(y)
\end{aligned}
$$

Therefore, $\lambda$ is an $L$-fuzzy prime ideal of $A$.

The converse of the above theorem is not true; for consider the given example below.

Example 4.4: Let $A=\{0, a, b, c\}, L=\{0, t, 1\}$ with $0<t<1$ and let $\vee$ and $\wedge$ be binary operations on $A$ defined by

\begin{tabular}{|c|c|c|c|c|}
\hline$\vee$ & $\mathbf{0}$ & $\mathbf{a}$ & $\mathbf{b}$ & $\mathbf{c}$ \\
\hline $\mathbf{0}$ & $\mathbf{0}$ & $\mathbf{a}$ & $\mathbf{b}$ & $\mathbf{c}$ \\
\hline $\mathbf{a}$ & $\mathbf{a}$ & $\mathbf{a}$ & $\mathbf{a}$ & $\mathbf{a}$ \\
\hline $\mathbf{b}$ & $\mathbf{b}$ & $\mathbf{b}$ & $\mathbf{b}$ & $\mathbf{b}$ \\
\hline $\mathbf{c}$ & $\mathbf{c}$ & $\mathbf{a}$ & $\mathbf{b}$ & $\mathbf{c}$ \\
\hline
\end{tabular}

\begin{tabular}{|c|c|c|c|c|}
\hline$\wedge$ & $\mathbf{0}$ & $\mathbf{a}$ & $\mathbf{b}$ & $\mathbf{c}$ \\
\hline $\mathbf{0}$ & $\mathbf{0}$ & $\mathbf{0}$ & $\mathbf{0}$ & $\mathbf{0}$ \\
\hline $\mathbf{a}$ & $\mathbf{0}$ & $\mathbf{a}$ & $\mathbf{b}$ & $\mathbf{c}$ \\
\hline $\mathbf{b}$ & $\mathbf{0}$ & $\mathbf{a}$ & $\mathbf{b}$ & $\mathbf{c}$ \\
\hline $\mathbf{c}$ & $\mathbf{0}$ & $\mathbf{c}$ & $\mathbf{c}$ & $\mathbf{c}$ \\
\hline
\end{tabular}

Then, $(A, \wedge, \vee, 0)$ is an ADL. Now define $\lambda: A \rightarrow L$ by $\lambda(0)=1$,

$\lambda(a)=\lambda(b)=0$ and $\lambda(c)=t$. Therefore, $\lambda_{0}=A, \lambda_{t}=\{0, c\}$ and $\lambda_{1}=\{0\}$ are prime ideals of $A$. Therefore, $\lambda$ is an $L$-fuzzy prime ideal of $A$, while $\lambda$ is not a prime $L$-fuzzy ideal of $A$, since $\lambda$ is not exactly two valued.

Finally, in this section we slightly generalize $\alpha$-level fuzzy ideals of $A$ and identify general prime ideals of $A$ with $L$-fuzzy prime ideals of $A$.

Theorem 4.5: Let $I$ a proper ideal of $A$ and $\alpha, \beta \in L$. Let $\langle\alpha, \beta\rangle_{I}$ be an $L$-fuzzy subset of $A$ defined by

$$
\langle\alpha, \beta\rangle_{I}(x)= \begin{cases}1 & \text { if } x=0 \\ \alpha & \text { if } 0 \neq x \in I \\ \beta & \text { if } x \notin I .\end{cases}
$$

Then,

(1) $\langle\alpha, \beta\rangle_{I}$ is an $L$-fuzzy ideal of $A$ if and only if $\beta \leq \alpha$ and, in this case $\langle\alpha, \beta\rangle_{I}$ is proper if and only if $\beta<1$. ideal of $A$

3) Suppose that 0 be a prime element in $L$. Then, $I$ is a prime ideal of $A$ if and only if $\langle\alpha, \beta\rangle_{I}$ is an $L$-fuzzy prime ideal of $A$ for all $1 \neq \beta \leq \alpha$ in $L$. cations.

Proof: (1) and (2) are striaght forward and simple verifi(3): Suppose that $I$ is a prime ideal of $A$ and $1 \neq \beta \leq \alpha$ in $L$. Let $x, y \in I$. Then, $x \wedge y=0 \Rightarrow x=0$ or $y=0$

$$
\Rightarrow\langle\alpha, \beta\rangle_{I}(x \wedge y)=1=\langle\alpha, \beta\rangle_{I}(x) \text { or }\langle\alpha, \beta\rangle_{I}(y)
$$

$0 \neq x \wedge y \in I \Rightarrow 0 \neq x \in I$ or $0 \neq y \in I$

$$
\Rightarrow\langle\alpha, \beta\rangle_{I}(x \wedge y)=\alpha=\langle\alpha, \beta\rangle_{I}(x) \text { or }\langle\alpha, \beta\rangle_{I}(y)
$$

and $x \wedge y \notin I \Rightarrow x \notin I$ and $y \notin I$

$$
\Rightarrow\langle\alpha, \beta\rangle_{I}(x \wedge y)=\beta=\langle\alpha, \beta\rangle_{I}(x)=\langle\alpha, \beta\rangle_{I}(y) .
$$

Converse follows from the fact that $\chi_{I}=\langle 1,0\rangle_{I}$. 


\section{Minimal PRIME L-FuZZY IDEALS}

Let us recall from [?] that a prime ideal $P$ an ADL of $A$ containing an ideal $I$ is said to be a minimal prime ideal belonging to $I$ if there is no prime ideal of $A$ containing $I$ and properly contained in $P$.

Definition 5.1: Let $\lambda$ be a prime $L$-fuzzy ideal of $A$. Then $\lambda$ is said to be minimal if $\lambda$ is a minimal member in the set of all prime $L$-fuzzy ideals of $A$ under the point-wise partial ordering. A minimal prime $L$-fuzzy ideal belonging to $\chi_{\{0\}}$ is simply called a minimal prime $L$-fuzzy ideal.

In this section, we characterize all minimal prime $L$-fuzzy ideals of $A$ in terms of minimal prime ideals of $A$ and minimal prime elements of $L$.

As usual, by a minimal prime element of $L$ we mean a minimal element in the poset of all prime elements of $L$.

Now we have the following:

Theorem 5.2: Let $\lambda$ be an $L$-fuzzy ideal of $A$. Then $\lambda$ is a minimal prime $L$-fuzzy ideal of $A$ if and only if $\lambda=\alpha_{I}$, for some minimal prime ideal $I$ of $A$ and a minimal prime element $\alpha$ in $L$.

Proof: Suppose that $\lambda=\alpha_{I}$ for some minimal prime ideal $I$ of $A$ and minimal prime element in $L$. Then by theorem 3.4, $\lambda$ is prime $L$-fuzzy ideal of $A$. Let $\mu$ be a prime $L$-fuzzy ideal of $A$ and $\mu \leq \lambda$. Then by theorem $3.4, \mu=\beta_{J}$ for some prime ideal $J$ of $A$ and a prime element $\beta$ in $L$. Therefore, $\beta_{J} \leq \alpha_{I}$. This implies that, $\beta \leq \alpha$ and $J \subseteq I$. By using the minimality of $I$ and $\alpha$, we get that $\beta=\alpha$ and $J=I$. Therefore, $\mu=\lambda$ and hence $\lambda$ is a minimal prime $L$-fuzzy ideal of $A$.

Conversely suppose that $\lambda$ is a minimal prime $L$-fuzzy ideal of $A$. Then by theorem 3.4 , there exists a prime ideal $I$ of $A$ and a prime element $\alpha$ in $L$ such that

$\lambda=\alpha_{I}$. Let $J$ be a prime ideal of $A$ such that $J \subseteq I$. Then $\alpha_{J} \leq \alpha_{I}$, by the minimality of $\lambda, \alpha_{J}=\alpha_{I}$. Therefore, $J=I$ and hence $I$ is minimal prime ideal of $A$. Let $\beta$ be a prime element in $L$ and $\beta \leq \alpha$. Then $\beta_{I} \leq \alpha_{I}$. This implies, $\beta_{I}=\alpha_{I}$ and hence $\beta=\alpha$. Thus $\alpha$ is a minimal prime element in $L$.

If the smallest element 0 in $L$ is prime, then 0 will be the only minimal prime element in $L$. Note that $\chi_{P}=0_{P}$, for any ideal $P$ of $A$.

The following is a simple verification.

Theorem 5.3: Let 0 be a prime element in $L$. Then an $L$ fuzzy ideal $\lambda$ of $A$ is a minimal prime $L$-fuzzy ideal of $A$ if and only if $\lambda=\chi_{P}$, for some minimal prime ideal $P$ of $A$. More over, $P \mapsto \chi_{P}$ is a bijection of the set of minimal prime ideals of $A$ onto the set of minimal prime $L$-fuzzy ideals of $A$.

\section{L-FuZZY Minimal PRIME IDEALS}

By an $L$-fuzzy minimal prime ideal of $A$ we mean, as usual, a minimal element in the set of all $L$-fuzzy prime ideals of $A$ under the point-wise partial ordering. In this section, we characterize all $L$-fuzzy minimal prime ideals of $A$ in terms of their $\alpha$-cuts.

Theorem 6.1: (1) If $\lambda$ is an $L$-fuzzy prime ideal of $A$, then $\lambda_{1}=\{x \in A: \lambda(x)=1\}$ is a prime ideal of $A$.
(2) Let $\lambda$ be an $L$-fuzzy prime ideal of $A$. If $\lambda$ is an $L$-fuzzy minimal prime ideal of $A$, then $\lambda_{1}$ is a minimal prime ideal of A.

Proof: (1) Let $\lambda$ be an $L$-fuzzy prime ideal of $A$. Then $\lambda_{1}$ is a proper ideal of $A$ since $\lambda$ is proper. Let $x, y \in A$. Then, $x \wedge y \in \lambda_{1} \Rightarrow \lambda(x \wedge y)=1$

$$
\begin{aligned}
& \Rightarrow 1=\lambda(x \wedge y)=\lambda(x) \text { or } \lambda(y) \text { (by } 4.1) \\
& \Rightarrow x \in \lambda_{1} \text { or } y \in \lambda_{1} .
\end{aligned}
$$

Thus, $\lambda_{1}$ is a prime ideal of $A$.

The converse is not true. For, consider the lattice $A=$ $\{0, a, b, c, 1\}$ represented by the Hasse diagram is given below.

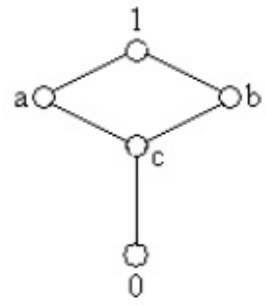

Define $\lambda: A \rightarrow[0,1]$ by $\lambda(0)=1, \lambda(c)=0.75, \lambda(b)=0.5$ and $\lambda(a)=\lambda(1)=0$. Then, $\lambda_{1}=\{0\}$ which is a prime ideal of $A$, while , $\lambda$ is not an $L$-fuzzy prime ideal of $A$, since $\lambda(a \wedge b)=\lambda(c)=0.75 \neq \lambda(a)$ and $\lambda(b)$.

(2) Suppose that $\lambda$ is an $L$-fuzzy minimal prime ideal of $A$. Let $Q$ be a prime ideal of $A$ and $Q \subset \lambda_{1}$. Then $\chi_{Q}$ is an $L$-fuzzy prime ideal of $A$ and $\chi_{Q} \supsetneqq \lambda$. This implies that $\lambda$ is not an $L$-fuzzy minimal prime ideal of $A$, which is a contradiction. Thus $\lambda_{1}$ is a minimal prime ideal of $A$.

The converse is not true; for in the above example, if $\lambda(0)=$ 1 and $\lambda(x)=0.5$ for all $x \neq 0$, then it can be easily checked that $\lambda$ is an $L$-fuzzy prime ideal of $A$ and $\lambda_{\alpha}=A$ if $0 \leq \alpha \leq 0.5$ and $\lambda_{\alpha}=\{0\}$ if $0.5<\alpha \leq 1$. In particular, $\lambda_{1}$ is a minimal prime ideal of $A$. But, $\lambda$ is not an $L$-fuzzy minimal prime ideal of $A$, since if we define $\mu(0)=1$ and $\mu(x)=0.25$ for all $x \neq 0$, then $\mu$ is an $L$-fuzzy prime ideal of $A$ and $\mu \supsetneqq \lambda$.

The following theorem is a characterization of $L$-fuzzy minimal prime ideals of $A$.

Theorem 6.2: Let $\lambda$ be an $L$-fuzzy prime ideal of $A$ and 0 be a prime element in $L$. Then $\lambda$ is an $L$-fuzzy minimal prime ideal of $A$ if and only if $\lambda_{\alpha}$ is a minimal prime ideal of $A$, for all $\alpha \in L$.

Proof: Suppose $\lambda$ is an $L$-fuzzy minimal prime ideal of $A$ and $\lambda_{\alpha}$ is not minimal prime ideal of $A$, for some $0<\alpha<1$ in $L$. Then there exists a prime ideal $Q$ of $A$ such that $Q \subset \lambda_{\alpha}$. Define $\mu: A \rightarrow L$ by

$$
\mu(x)= \begin{cases}1 & \text { if } x=0 \\ \alpha & \text { if } 0 \neq x \in Q \\ 0 & \text { if } x \notin Q .\end{cases}
$$

Then, clearly $\mu=(\alpha, 0)_{Q}$ and hence $\mu$ is an $L$-fuzzy prime ideal of $A$ (by theorem 4.5 (3)). Also, $\mu \leq \lambda$. Since $Q \subset \lambda_{\alpha}$, there exists $y \in \lambda_{\alpha}$ such that $y \notin Q$. Therefore, $\mu(y)=0<$ 
$\alpha \leq \lambda(y)$. Therefore, $\mu \supsetneqq \lambda$, which is a contradiction. Thus for each $\alpha \in L, \lambda_{\alpha}$ is a minimal prime ideal of $A$.

Conversely, suppose for each $\alpha \in L, \lambda_{\alpha}$ is a minimal prime ideal of $A$. Let $\mu$ be an $L$-fuzzy prime ideal of $A$ such that $\mu \leq \lambda$. Then for each $\alpha \in L, \mu_{\alpha} \subseteq \lambda_{\alpha}$. By the minimality of $\lambda_{\alpha}$, we have $\mu_{\alpha}=\lambda_{\alpha}$ and hence $\mu=\lambda$. Therefore $\lambda$ is an $L$-fuzzy minimal prime ideal of $A$.

Remark 6.3: If $\lambda$ is an $L$-fuzzy minimal prime ideal of $A$, the each $\alpha$-cut of $\lambda$ need not be minimal prime ideal of $A$.

For, consider the example given in the following. Let $A=$ $\{0, a, b, c, 1\}$ be the lattice represented by the Hasse diagram is given below.

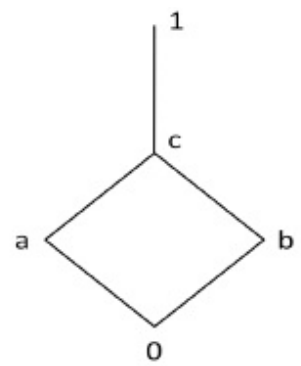

Define $\lambda: A \rightarrow[0,1]$ by $\lambda(0)=\lambda(a)=1$, $\lambda(b)=\lambda(c)=0.5$ and $\lambda(1)=0$. It can be easily verified that, $\lambda$ is an $L$-fuzzy prime ideal of $A$ and for any $t \in[0,0.5]$, $\lambda_{t}=\{0, a, b, c\}$ is a prime ideal of $A$ but not minimal.

\section{REFERENCES}

[1] L. Zadeh, "Information and control," Fuzzy sets, vol. 8, no. 3, pp. 338353, 1965.

[2] J. Goguen, "L-fuzzy sets," Journal of mathematical analysis and applications, vol. 18, no. 1, pp. 145-174, 1967.

[3] W.-j. Liu, "Fuzzy invariant subgroups and fuzzy ideals," Fuzzy sets and systems, vol. 8, no. 2, pp. 133-139, 1982.

[4] T. Mukherjee and M. Sen, "On fuzzy ideals of a ring i," Fuzzy Sets and systems, vol. 21, no. 1, pp. 99-104, 1987.

[5] U. Swamy and K. Swamy, "Fuzzy prime ideals of rings," Journal of Mathematical Analysis and Applications, vol. 134, no. 1, pp. 94-103, 1988.

[6] U. Swamy and G. Rao, "Almost distributive lattices," Journal of the Australian Mathematical Society, vol. 31, no. 1, pp. 77-91, 1981.

[7] U. Swamy, C. S. S. Raj, and N. Teshale, "Fuzzy ideals of almost distributive lattices," Annals of Fuzzy Mathematics and Informatics, vol. 14, no. 4, pp. 371-379, 2017. 